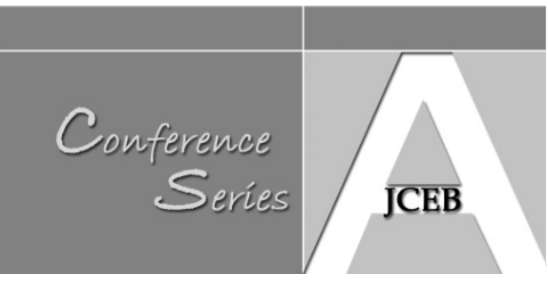

\title{
A Passive Rotary System for Seismic Risk Mitigation of Steel Structures
}

\author{
Ricky Chan, Peter S.P. Wong (RMIT University, Australia)
}

\begin{abstract}
This paper presents a novel bracing system designed for earthquake risk mitigation for steel structures. It involves a rotary system which a Chebyshev linkage connected to the ground and the building frame. Upon earthquake excitation, movement of structure floor slabs causes a rotational motion in the disc. Displacement-based dampers are installed between the rotary system and the ground which damp the structural vibrations. The system amplifies the travel of the dampers and efficiency is enhanced. In addition, the cross-brace members are always in tension, permitting the use of very slender sections. The paper first reviews the governing equations of the system, followed by a physical model demonstration. A 3-degreeof-system model with the proposed rotary system was subjected to simulated ground shaking. Acceleration on top floor was measured. Results demonstrated that proposed system effectively supresses the vibrational characteristics of the structure, and represents a viable and inexpensive solution to mitigate seismic risks.
\end{abstract}

Keywords: Earthquake engineering, passive energy dissipation

\section{Introduction}

In the last few decades significant research and development has been made to make civil structures safer and more robust with the use of energy dissipating systems (Symans et al., 2008). In this technique designated energy dissipating devices (EDD) are in-stalled in the structure such that a portion of the energy originated from ground shaking is diverted to. Among many EDDs developed, dampers that utilize metallic yielding to dissipate energy are widely adopted for they are inexpensive, insensitive to frequency of vibration, and require minimal maintenance. Developed metallic EDD include the patented ADAS (Bergman et al 1987), its variant triangular shape TADAS and the Steel Slit Damper (Chan and Albermani, 2008). The Buckling-restrained brace (Black et al., 2004) and its variant Buckling-restrained lug (Chan and Albermani, 2013), on the other hand, makes use of the axial deformation of steel. Shear yielding of thin steel plates have also been researched (Chan et al., 2009). Researchers have also been made use of friction as a method to dissipate input energy. Friction dampers utilize the mechanism of solid friction that develops between two solid surfaces sliding relative to one another is proven to be effective in laboratories. Several types of friction dampers have been developed. The Slotted Bolted Connection (Grigorian et al., 1993) is a simple yet effective device which makes use of the friction between two metallic surfaces. Generally, a good displacement-based device for seismic applications must exhibit: (1) adequate elastic stiffness to withstand in-service lateral loads (e.g. wind); (2) the yield strength of the damper exceeds the expected in-service lateral loads such that the device is activated only in an earthquake event; (3) large energy dissipative capability; and (4) stable hysteretic force-displacement response which can be modelled numerically.

\section{Magnification Factor}

A main difficulty concerning the application of displacement-based passive system is, energy dissipation of dampers relies on sufficient damper travel during excitation. When such displacements occur in dampers the main structural elements may have been damaged. It is particularly problematic for structures which possess limited ductility, such as reinforced concrete frames. To magnify the damper travels, various approaches have been suggested. One popular approach is to install dampers in toggle-damper-brace systems. Constantinou 
et al. (2001) defined a magnification factor which is expressed by the ratio between damper travel and storey displacement, i.e.

$$
f=\frac{u_{d}}{u}
$$

With $f$ values larger than unity, the damper travel is magnified. Magnifying the damper travel reduces the damper force. $f$ values for upper, lower and reverse toggle arrangement has been formulated (Constantinou et al., 2001) and it has been concluded that practical range of $f$ values are between 2.0-5.0. A single-storey frame with upper toggle configuration with $f$ approximately 3.0 has been tested in experiments. Another approach to amplify damper travel includes the scissor-jack configuration (Slgaher and Constantinou, 2003). The toggledamper-brace system generally requires braces which are stiff enough to elude compression buckling. A double upper-toggle-brace system has been proposed for low-rise structures which suffer from the soft-storey mechanism (Chan et al., 2012).

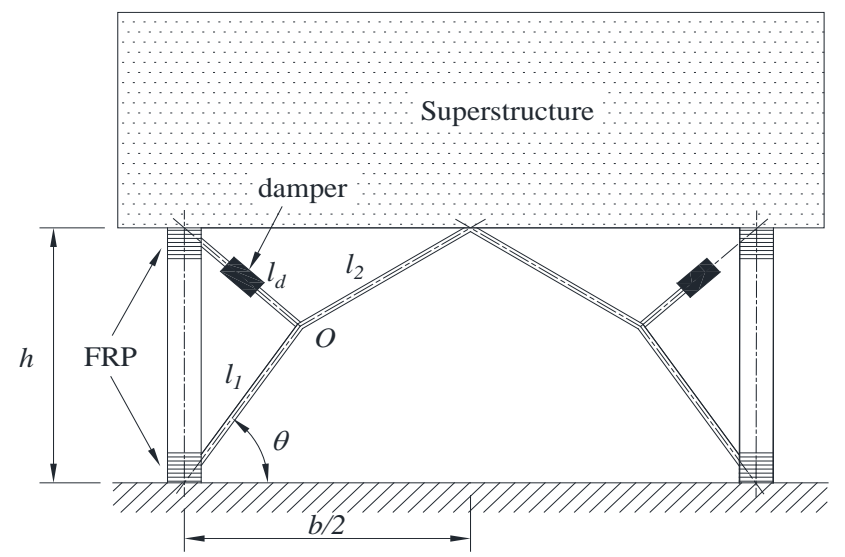

Figure 1. Double upper-toggle-brace damper arrangement (Chan et al., 2012)

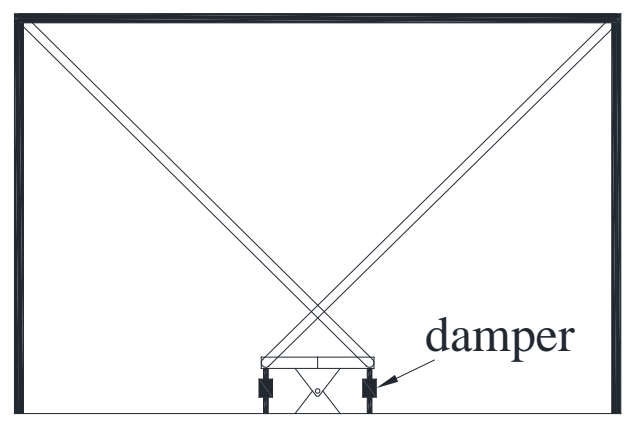

Figure 2. The seesaw system (Tagawa and Gao, 2012)

On the other hand, Professor $\mathrm{H}$. Tagawa of Hiroshima University recently proposed a system called Seesaw System in which a Chebyshev linkage is incorporated with dampers (Figure 2) (Tagawa and Gao, 2012). Upon earthquake excitation, movement of structure causes a rocking motion in the seesaw. Dampers are installed underneath the seesaw which damp down the structural vibrations. Similar to the toggle configuration, the Seesaw System amplifies the travel of the dampers; as a result the efficiency of the dampers is enhanced. Through pre-tensioning of the braces, the braces can be kept in tension throughout design displacement, thus compression buckling of the brace members are eluded and allowing a small cross section to be used. A metallic U-damper has been specifically designed for the 
Seesaw System. Magnification factor as a function of seesaw geometry has been reported. More recently, studies have been made to the seesaw system using velocity-dependent typical viscoelastic damper (Kang and Tagawa, 2013).

\section{Proposed Rotary System}

The seesaw system tends to cause significant strength demand onto the seesaw beam. In view of this concern a novel system is proposed herein, namely the Passive Rotary System. Figure 3 presents a schematic drawing of the rotary system in its un-deformed shape. The system is characterised by mass $m$, horizontal elastic stiffness $k$ and damping ratio $c$. A rotary disc of diameter $r$ with its centre secured at a position $y$ above ground. Two brace members connect the eaves of the storey to point $E$ near the circumference of the disc. The length of the brace members and the angle between the brace and vertical is denoted by $l$ and $\varphi$ respectively.

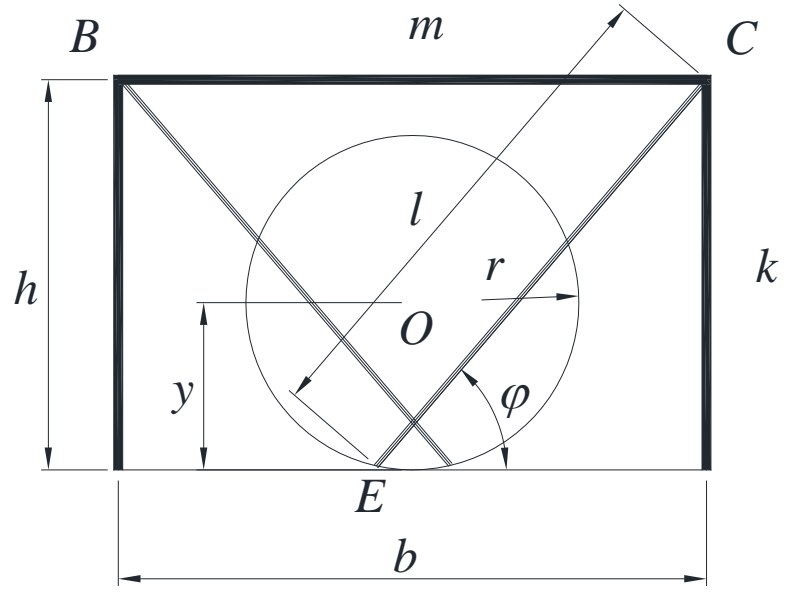

Figure 3. The passive rotary system: un-deformed shape

As shown in Figure 4, upon horizontal displacement of storey $u$ to the right, the brace in tension pulls the disc to rotate through an angle $\theta$. The new position of $E$ becomes $E$ '. If a displacement-based damper is connected between a point on the disc circumference and fixed point of ground, this damper undergoes a displacement. Hence, the magnification factor can be described as,

$f=\frac{r \theta}{u}$

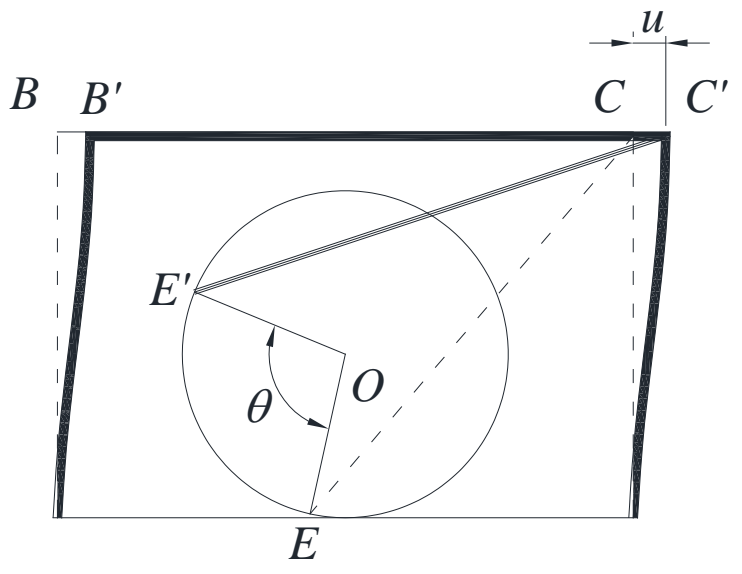

Figure 4. The passive rotary system: deformed shape

Chan, R \& Wong, P.S.P. 2014, 'A Passive Rotary System for Seismic Risk Mitigation of Steel Structures', Australasian Journal of Construction Economics and Building Conference Series, 2(2), 72-79. 
To obtain the geometric relationship between building dimensions $h$ and $b$, the brace length $l$ and angle $\varphi$, we first make an assumption which the brace is axially rigid. When the storey is displaced by $u$, the position of $E$ ' may be easily calculated by intersection of two circles: the first circle is centred at $O$ and has radius $r$, and the second circle is centred at $C$ ' with radius equals to $l$. $E$ ' is one of the intersections between the two circles.

In particular, if we define the position $E$ by the angle from vertical, denoted by $\tau$, it can be shown that the geometry of the system is completely defined by only five parameters: $h, b, r$, $y$ and $\tau$. If building dimensions $h$ and $b$ are fixed by the building owner or the architect, design engineer only chooses three parameters: $r, y$ and $\tau$.

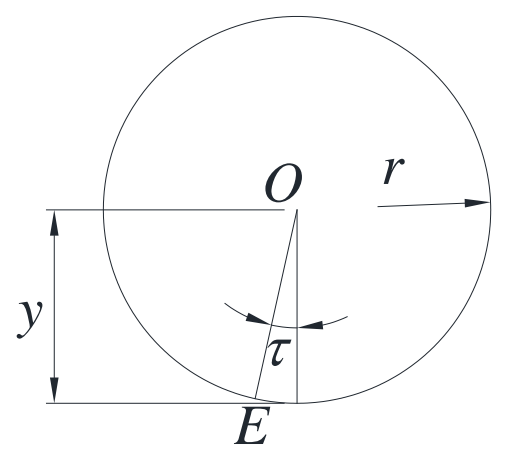

Figure 5. Three parameters required to define rotary system

For a single storey building with $h=3.5 \mathrm{~m}$ and $b=6.0 \mathrm{~m}$, the magnification factor $f$ with relation to other geometric parameters are shown below. Figure 6 shows the relationship between $f$ and $\tau$ with various disc elevations. The radius of disc is fixed at $0.5 \mathrm{~m}$. The figure suggests that very large magnification factor can be achieved. The relationship between $f$ and $\tau$ is nonlinear. It also shows that $f$ increases with elevation of disc $y$ increases. The figure suggests a magnification factor of approximately 20 can be achieved over practical ranges of $\tau$.

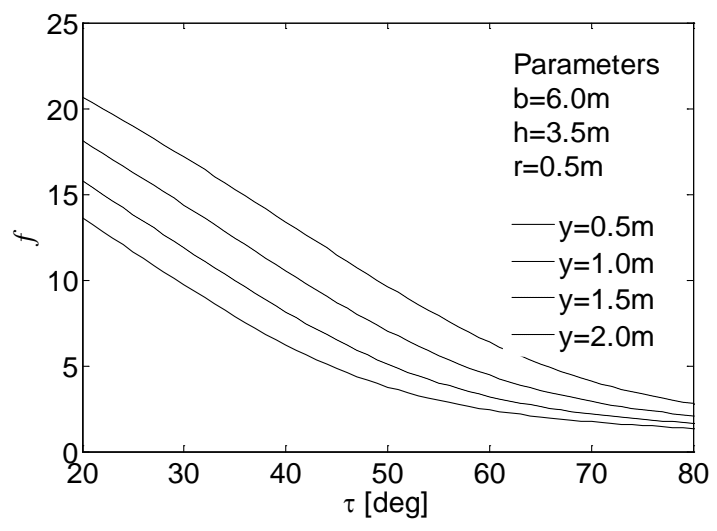

Figure 6. Magnification factor for various disc elevation

Figure 7 shows the relationship between $f$ and $\tau$ with various disc radii. Elevation of disc centre is arbitrarily selected at mid-height. The figure suggests that a larger disc will result in larger magnification factors. With a small $\tau$ angle a magnification factor may increase up to 80.

Chan, R \& Wong, P.S.P. 2014, ‘A Passive Rotary System for Seismic Risk Mitigation of Steel Structures’, Australasian Journal of Construction Economics and Building Conference Series, 2(2), 72-79. 


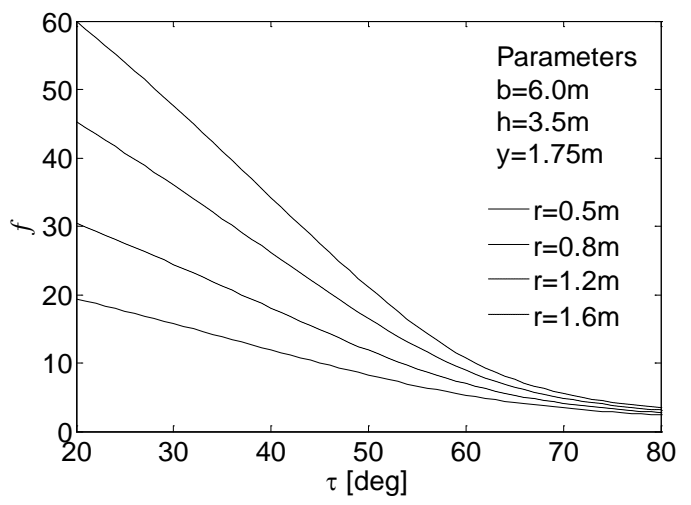

Figure 7. Magnification factor for various disc radii

It is also possible to apply the rotary system in a multi-storey building, as shown in Figure 8. The roof level is connected to a rotary disc located in the lowest level by a pair braces members. As the system only requires one tension brace to activate the disc, very slender braces such as cable elements will be sufficient. The magnification factor, however, will be affected by the geometry. The relationship between $\tau$ and the disc radii for a 3-level structure is shown in Figure 9. It can be observed that the magnification factor has greatly reduced compared to the single storey cases. A larger disc radius still produces a larger $f$ but its effect is less prominent. When increases beyond approximately 40 degrees the value drops below unity. In other words the rotary system is ineffective in magnifying the damper travel.

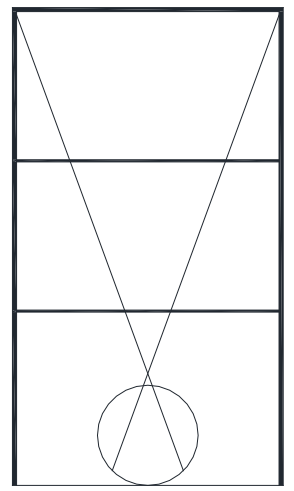

Figure 8. Multi-level application

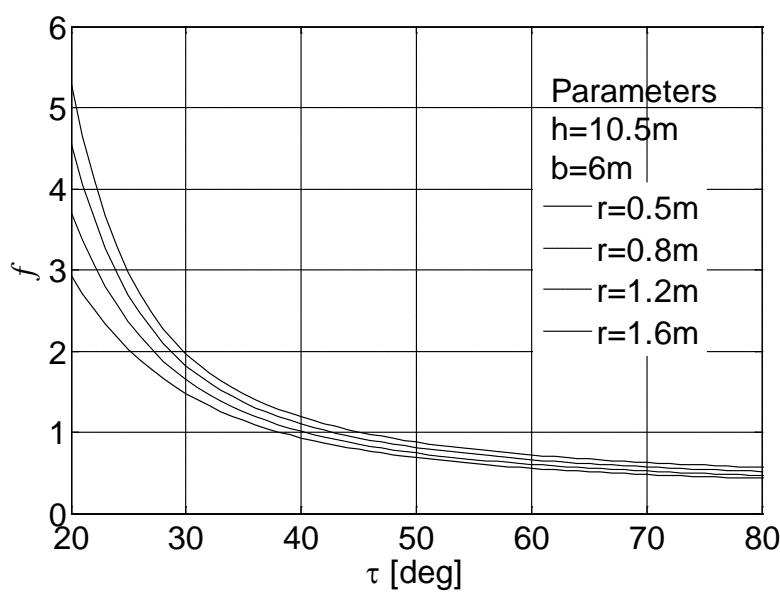

Figure 9. Magnification factor for a 3-level building

Chan, R \& Wong, P.S.P. 2014, 'A Passive Rotary System for Seismic Risk Mitigation of Steel Structures’, Australasian Journal of Construction Economics and Building Conference Series, 2(2), 72-79. 


\section{Physical Model Demonstration}

A 3-storey model frame is built as a physical demonstration of the concept. Ground motion is simulated by an electric belt-driven slider which is capable of produce repetitive linear motions. Due to the payload limitation of the electric slider a light-weighted model frame is warranted. Columns are made of $25 \mathrm{~mm} \times 1 \mathrm{~mm}$ aluminium strips, while floor plates are made from $3 \mathrm{~mm}$ thick Plexiglas material. Connections are made from aluminium brackets and steel $3 \mathrm{~mm}$ diameter steel bolts and nuts. A block of wood which weighs $0.35 \mathrm{~kg}$ is placed on the top level. Figure 10 shows an overview of the model frame. Free-vibration test of the model frame with brace member detached from the rotary disc indicates a first mode period of 0.35 second. The rotary system comprises of a $5 \mathrm{~mm}$ thick, $150 \mathrm{~mm}$ diameter plastic disc. The centre of the disc is located at $100 \mathrm{~mm}$ from the base. A pair of cross-braces connects the top level to the rotary disc. The brace members are made of $12 \mathrm{~mm} \times 3 \mathrm{~mm}$ aluminium bars. The excitation is generated by an electric slider. An accelerometer is placed at the top level to monitor the excitation of the frame. A 20-cycle motion is selected. Measured peak acceleration of the slider is $0.56 \mathrm{~g}$ and a forcing period of 0.4 second. No specific damper is installed since the inherent friction between contacting surfaces in the model, particularly between the touching brace members, already provided a good amount of energy dissipating capability.

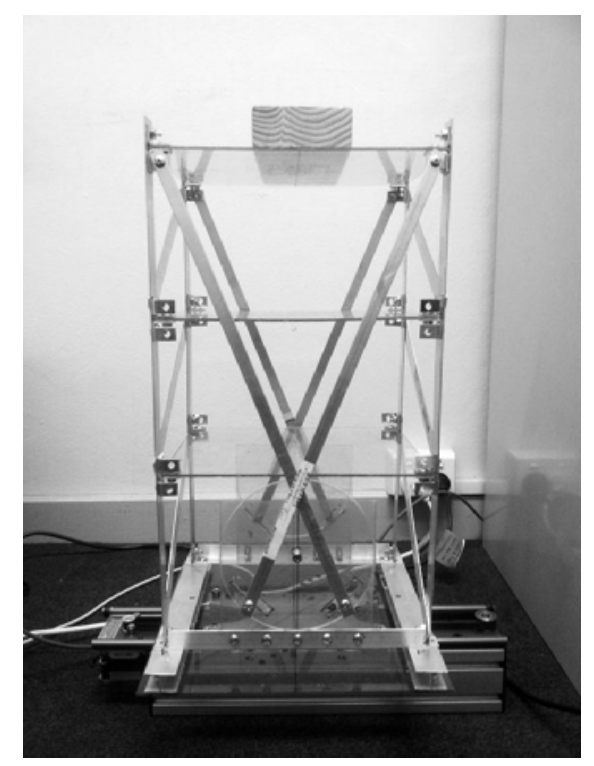

Figure 10. Overview of experimental setup

\section{Observations}

The first test involves the model frame with the brace detached from the disc in order to maintain the same overall weight. This un-braced frame is observed to vibrate in the first mode, and measured peak acceleration is $1.15 \mathrm{~g}$. When excitation has ceased the frame vibrates freely for about 10 seconds. A second test is run with the brace members connected to the rotary disc. During excitation the rotary system rotates in both directions as the braces pushes and pulled upon it. The measure roof acceleration has dramatically decreased to approximately $0.55 \mathrm{~g}$. Measured acceleration $\mathrm{n}$ the roof-level is plotted in Figure 11. 


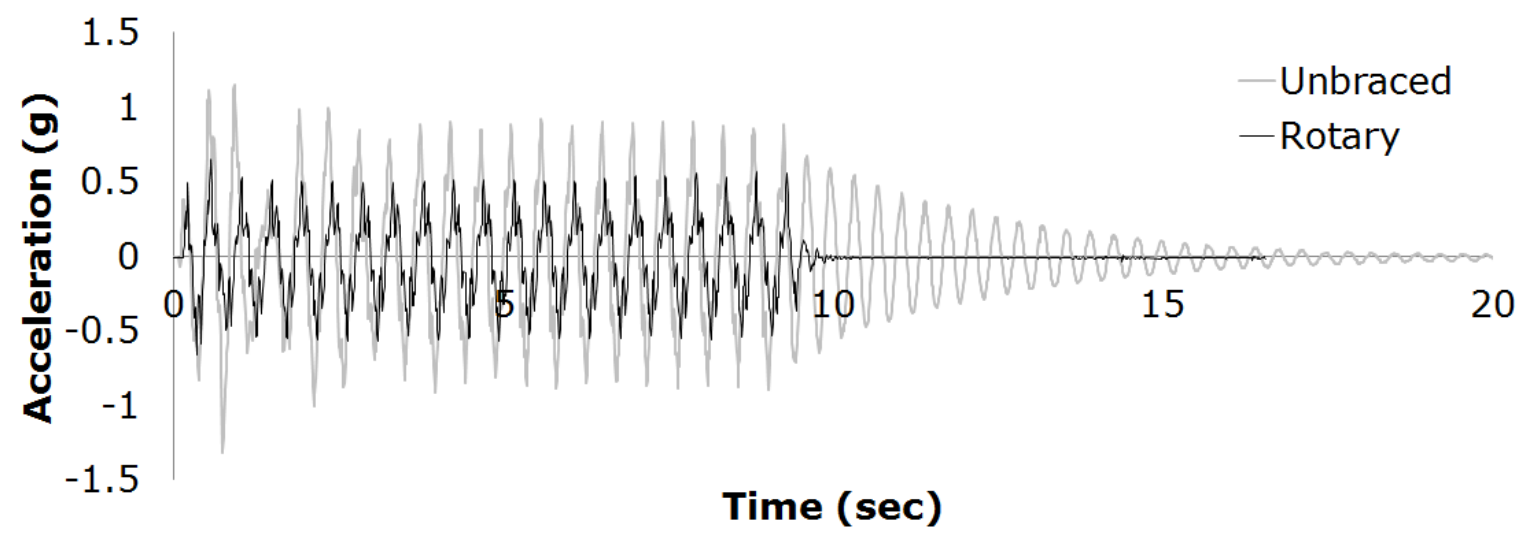

Figure 11. Measured roof-level acceleration

\section{Conclusion}

This article presents a novel bracing system for the earthquake-risk mitigation of building frames. It involves a pair of brace members and a rotary disc forming a Chebyshev linkage. The mechanism converts horizontal displacement of the storey into rotational movement of the disc. If a displacement-based energy dissipation device such as a friction damper is installed between a stationary point on the ground and the circumference of the disc, input energy from ground shaking can be dissipated, hence the effective damping of the structure is increased. This rotary system also amplifies the travel of the damper by a magnification factor $f$. Since dissipated energy (hence effective damping ratio) is proportional to the displacement travelled by the damper, magnifying the damper travel will enhance damper effectiveness. Relationship between $f$ and other geometrical parameters have been identified. To illustrate the proposed system in a dynamic situation a physical model demonstration is also presented in this article in which a lightweight aluminium frame is tested with and without the rotary system. The demonstration shows that the rotary system significantly reduces the acceleration of the roof level of the frame under ground shaking.

\section{References}

Black, C., Makris, N. \& Aiken, I. 2004. Component Testing, Seismic Evaluation and Characterization of Buckling-Restrained Braces. Journal of Structural Engineering, 130(6), 880-894.

Chan, R. \& Albermani, F. 2013. Buckling-restrained-lug connection for energy dissipation. Advances in Structural Engineering, 16(1), 11-20.

Chan, R. W. K. \& Albermani, F. 2008. Experimental study of steel slit damper for passive energy dissipation. Engineering Structures, 30 (4), 1058-1066.

Chan, R. W. K., Albermani, F. \& Williams, M. S. 2009. Evaluation of yielding shear panel device for passive energy dissipation. Journal of Constructional Steel Research, 65(2009), 260-268.

Constantinou, M., Tsopelas, P., Hammel, W. \& Sigaher, A. 2001. Toggle-Brace-Damper Seismic Energy Dissipation Systems. Journal of Structural Engineering, 127(2), 105-112.

Grigorian, C. E., Yang, T. S. \& Popov, E. P. 1993. Slotted Bolted Connection Energy Dissipators. Earthquake Spectra, 9(3), 491-504.

Kang, J. D. \& Tagawa, H. 2013. Seismic response of steel structures with seesaw systems using viscoelastic dampers. Earthquake Engineering and Structural Dynamics, 42(5), 779-794. 
Slgaher, A. N. \& Constantinou, M. C. 2003. Scissor-Jack-Damper Energy Dissipation System. Earthquake Spectra, 19(1), 133-158.

Symans, M., Charney, F., Whittaker, A., Constantinou, M., Kircher, C., Johnson, M. \& Mcnamara, R. 2008. Energy Dissipation Systems for Seismic Applications: Current Practice and Recent Developments. Journal of Structural Engineering, 134(1), 3-21.

Tagawa, H. \& Gao, J. 2012. Evaluation of vibration control system with U-dampers based on quasilinear motion mechanism. Journal of Constructional Steel Research, 70(2012), 213-225. 